\title{
An Efficient Clusterhead Election Algorithm for Mobile Ad-Hoc Network
}

\author{
Deepti Kothari \\ Research Scholar \\ Information Technology \\ M.I.T.M. Indore
}

\author{
D.Srinivasa Rao \\ Associate Professor \\ Computer Science \& \\ Engineering Department
}

\author{
Anil Singh \\ Associate Professor \\ Computer Science \& \\ Engineering Department
}

\begin{abstract}
Mobile ad hoc network, abbreviated as MANET, is flexible, self-configuring, wireless ad-hoc network. MANET do not require pre-existing or pre-defined infrastructure, instead it configures and creates a network dynamically. All available nodes (devices) in MANET act as routers and are allowed to move freely in any direction, even in and out of network. Communication in a network with such high levels of nodal mobility is complex and challenging.

The paper proposes an efficient weighted cluster election algorithm (WCA) by leveraging most connected, least utilized node for cluster head election. A comparative study of the traditional weighted cluster algorithm (WCA) and the proposed weighted cluster algorithm (WCA) simulation results shown significant improvement in performance, scalability, stability and efficiency of the network.
\end{abstract}

\section{Keywords}

Mobile ad hoc networks, Clustering, Throughput, Packet delivery Ratio, End to End delay.

\section{INTRODUCTION}

Mobile ad hoc network is a new generation network technology. In this network a group of network devices interact with each other for cooperative communication. In order to meliorate the performance of the network the clustering concept played essential role. Weighted Cluster Algorithm is widely accepted and most explored method for identifying efficient clusters.

At present, wireless cellular networks are more relied on wired set-up by which all base stations are connected, suggesting that networks are not flexible and constrained to a geographical area with a pre-defined boundary. Implementation of such networks is time consuming and cannot be relied in case of emergency. Therefore, mobile peer-to-peer networks play a critical role in scenarios where a wired (central) backbone is not economical to set-up, such as law enforcement operations, battlefield communications, disaster recovery situations, and so on. Such situations demands for a network where all the nodes including the base stations are mobile in nature, and communication must be supported undisturbed between any two nodes. Multi-cluster, multi-hop network should be able to configure itself for dynamic network.

\section{BACKGROUND}

Clustering is division of the network into different virtual groups, based on rules in order to discriminate the nodes allocated to different sub-networks. The goal is to achieve scalability in presence of large networks and high mobility. Properties of clustering are Geographically allocated, Balance resource use, Service localization, Virtual communication backbone allows inter-cluster communication that can be restricted to cluster-heads and cluster-gateways Cluster-Head is local coordinator of a cluster therefore update and maintain cluster information only locally and also minimize information stored and propagated in the network, cluster member is ordinary node, cluster-gateway is node with intercluster links, forwards information between cluster.

Nodes perform route discovery and maintenance. Flat routing works fine for small networks but not work in large MANETs. In proactive routing messages communication overhead occur; while in reactive routing high overhead occur just from route discovery. Hierarchical routing may work fine for large networks due to localized route search and information dissemination, communication flows follow hierarchical structures (i.e. social and organizational).

Authors classify clusters as DS (dominant set)-based clustering route maintenance actions to the nodes from the dominating set. Mobility aware clustering cluster on the mobility behaviour of the mobile node. Energy-efficient clustering considers the energy available at the nodes. Loadbalancing clustering limits the number of nodes in a cluster in order to distribute the work load. Combined-metrics clustering consider multiple metrics. Low-maintenance clustering performs clustering for upper-layers and reduces the maintenance cost.

Weighted Cluster Algorithm (WCA) computes the combined weight for a particular node by considering the connectivity of each node (number of nodes), sum of distances with neighbouring node, residual energy and mobility (defined as running average of speed).

\section{SOLUTION DOMAIN}

In order to find the stable and more efficient network cluster the following solutions are suggested to implement.

1. Energy: The node which having high remaining battery power have long life and able to participate in communication.

2. Lowest sequence number: In order to find most recent routing information in network, that is required to have lowest sequence number.

3. Connectivity: Maximum number of node connected to a node has more serving capability.

4. Buffer Length: Buffer length demonstrate the low load on node, therefore less loaded node can serve better.

5. Mobility: Low mobile nodes are able to form more stable clusters.

\section{SIMULATION SCENARIOS}

The simulation of the desired network clustering scheme is given using two different network simulation scenarios. 
Simulation using Traditional WCA Algorithm: In this simulation, previously available weighted clustering scheme is implemented using network simulator 2 and performance of the routing protocol is estimated.

Simulation using Proposed WCA Algorithm: In this simulation scenario proposed WCA algorithm is implemented and performance of the algorithm is compared with the traditional WCA Algorithm.

Simulation using NS2 consists of three main steps:

- To clearly specify the objectives and assumptions of the simulation, first the simulation must be designed.

- Secondly, to implement the concept designed in the first step it requires configuring and running simulation scenario.

- Final step is to collect the simulation result and trace the simulation if necessary.

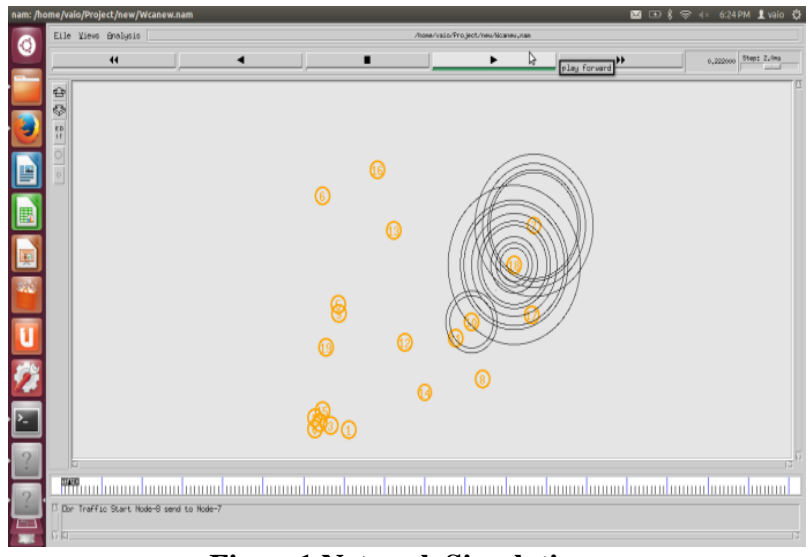

Figure1 Network Simulation

\section{RESULTS AND ANALYSIS}

The given section provides the detailed discussion about the network simulation and shows the comparison of the traditional and the proposed WCA algorithm.

\subsection{Throughput}

Network throughput is the degree of the message delivered successfully in a communication medium. This data may be carried in a physical or logical connections, or go through using a specific network device. The throughput is basically calculated in terms of bits per second and occasionally in terms of data packets per time slot or data packets per second.

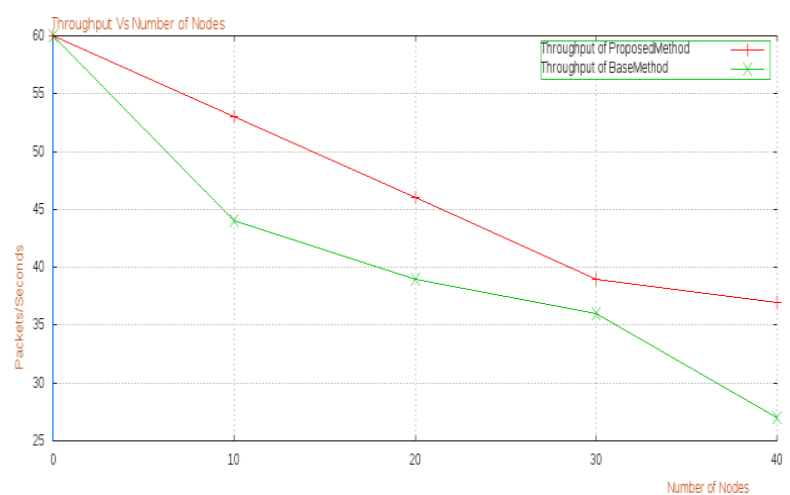

Figure2 Comparative Throughput

The figure 2 shows the comparative throughput using both the techniques. The red line provides the throughput of proposed WCA algorithm and the green line shows the previously available WCA algorithm the comparative performance according to the above given diagram is higher than previously available technique. In addition of that with increasing traffic the performance of network is shown in Figure3, this figure also demonstrate the effective results as compared to the traditional WCA approach.

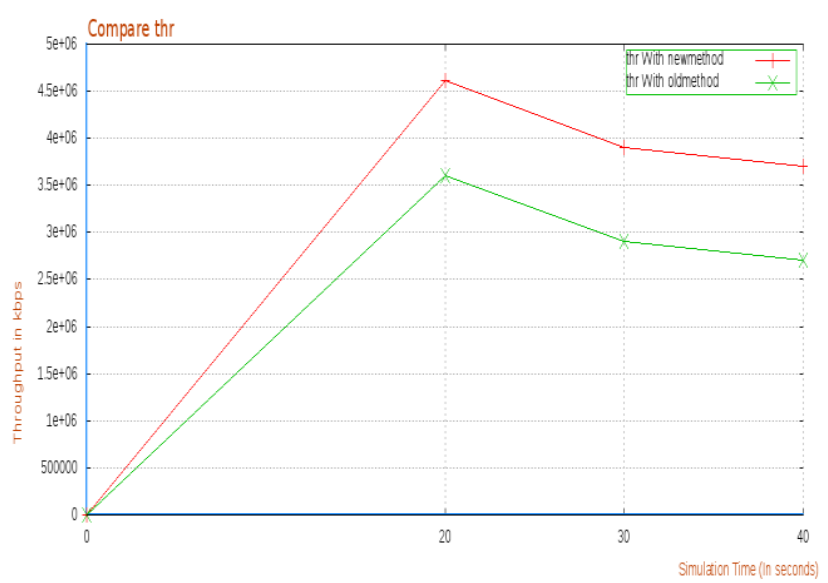

Figure3 Comparative Throughput

\subsection{2 PDR}

The amount of packet successfully delivered at the target network device is known as the packet delivery ratio.

$$
\text { packet delivery ratio }
$$$$
=\frac{\text { Total amount of delivered packets }}{\text { total sent packets }} \times 100
$$

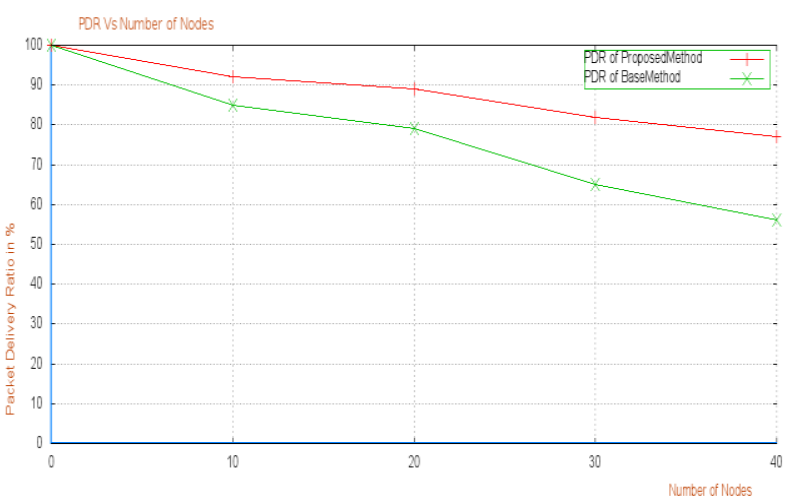

Figure4 Comparative PDR

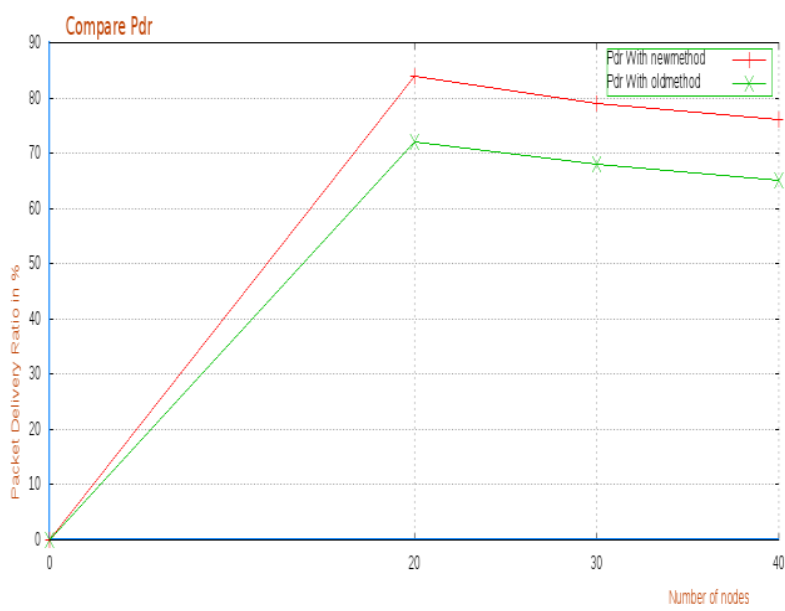

Figure5 Comparative PDR

Figure 4 shows the comparative packet delivery ratio in terms of percentage, the $\mathrm{Y}$ axis of the Figure shows the percentage 
packet delivery ratio and $\mathrm{X}$ axis shows the simulation time in seconds. According to the Figure4 the PDR ratio of the proposed weighted clustering algorithm is higher than the traditional WCA algorithm. Additionally in the Figure5 with increasing number of nodes the performance of network is evaluated. The proposed WCA algorithm outperforms with respect to the traditional WCA algorithm

\subsection{End to End Delay}

The total amount of time required to deliver a data packet to a targeted network device is known as the end to end delay. The comparative performance of both the WCA algorithm is shown in Figure6 and in Figure7. With increasing number of nodes the comparative performance is shown in Figure7, according to the obtained results the end to end delay of the proposed WCA algorithm is less than the previously available WCA algorithm.

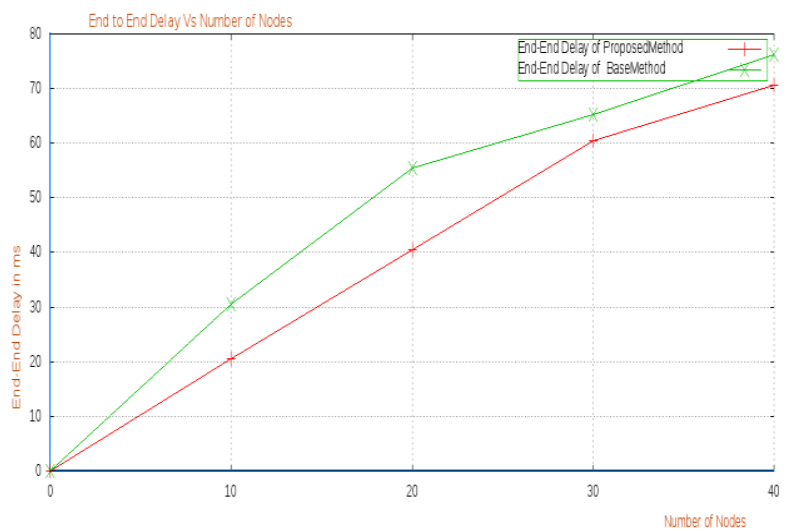

Figure6 Comparative End to End Delay

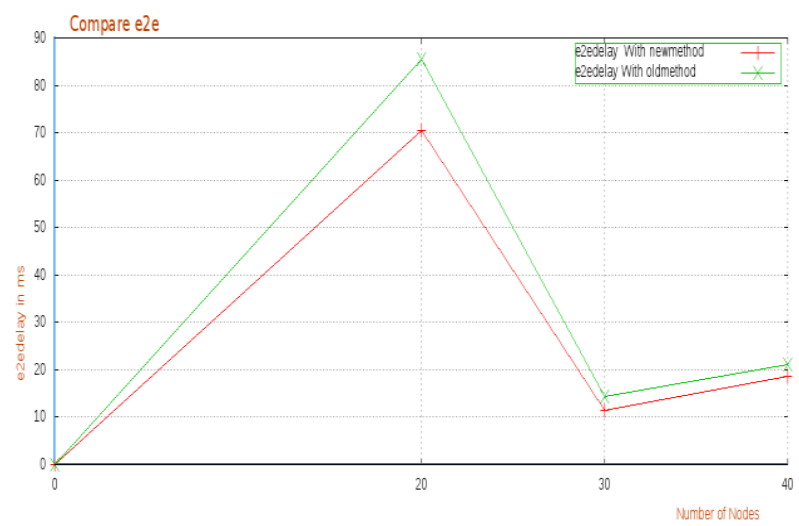

Figure7 Comparative End to End Delay

\section{CONCLUSION}

In this presented study mobile ad hoc networks and their challenges to find the more stable and efficient network clustering algorithms is explored and investigated. For that purpose various recently developed weighted clustering and optimum clustering schemes are learned. After investigation weighted clustering algorithm is modified with additional network parameters. Traditionally the weighted clustering algorithm is developed using the remaining energy, mobility, connectivity and available memory. This technique is further explored and enhanced using introducing the additional parameter in the WCA algorithm. In order to enhance the given technique lowest sequence number is added with the traditional WCA algorithm.

The implementation of the proposed WCA algorithm is done using TCL script development and simulation is performed over the NS2 (network simulator 2). After successful implementation of the traditional and proposed WCA algorithm, the performance of both algorithms is compared using different performance parameters. The obtained performance outcomes are summarized using table

Table1 Summary Table

\begin{tabular}{|c|c|c|c|}
\hline S.No. & Parameters & $\begin{array}{c}\text { Proposed } \\
\text { WCA }\end{array}$ & Traditional WCA \\
\hline 1 & Throughput & High & Low \\
\hline 2 & PDR & High & Low \\
\hline 3 & $\begin{array}{c}\text { End to End } \\
\text { Delay }\end{array}$ & Low & High \\
\hline
\end{tabular}

According to the simulation outcomes and the above listed summarized results the performance of the proposed WCA is optimum with respect to the traditional WCA, due to their high throughput, high packet delivery ratio and less end to end delay in network

\section{REFERENCES}

[1] HuiCheng and Shengxiang Yang, "Hyper-mutation based Genetic Algorithms for Dynamic Multicast Routing Problem in Mobile Ad-hoc Networks," in IEEE International Conference on Trust, Security, Privacy in Computing and Communication, Liverpool, 2012.

[2] Sung-Ju-Lee and Elizabeth M. Belding-Royer, "Scalability study of the ad hoc on-Demand Distance Vector Routing Protocol," in International Journal of Network Management; 2003, pp. 97-114

[3] Kapang Lego and Pranav Kumar Singh, "Comparative Study of Ad-hoc Routing Protocol AODV, DSR and DSDV in Mobile Ad-hoc Network," in Indian Journal of Computer Science and Engineering, vol 1, no. 4, pp. 364371.

[4] Shiva Prakash. P. Saini and S. C. Gupta, "Methodologies and Applications of Wireless Mobile Ad-hoc Networks Routing Protocols," in International Journal of Applied Information System, Newyork, 2012, pp. 5-15.

[5] TanuPreet Singh and Neha "Multicast Routing Protocols in Manet," in International Journal of Advanced Research in Computer Science and Software Engineering, vol.2, January 2012.

[6] E. Bommaiahand M. Liu "AMRoute: Ad-hoc Multicast Routing Protocol", in Internet-Draft, August 1998.

[7] C. W. Wu and C. K. Toh et al, "Ad-hoc Multicast Routing Protocol Utilizing Increasing id-numbers (AMRIS) Functional Specification," in Internet-Draft, November 1998.

[8] Naveen Chauhan and Lalit Kumar Awasthi "A Distributed Weighted Cluster Based Routing Protocol for MANETs," in Scientific Research on Wireless Sensor Network, 2011, pp. 54-60.

[9] Mainak Chatterjee and Sajal K. Das"WCA: A Weighted Clustering Algorithm for Mobile Ad Hoc Networks," in Cluster Computing, Netherland, 2002, pp. 193-204. 
[10] Noman Mohammed and HadiOtrok "Mechanism DesignBased Secure Leader Election Model for Intrusion Detection in MANET," in IEEE Transactions On Dependable and Secure Computing, 2009, pp.1-15.

[11] Ditipriya Sinha and Uma Bhattacharya et al, "CLAR: A Novel Cluster Oriented Agent Based Routing Protocol for Manet," in Foundation of Computing and Decision Sciences, 2013.

[12] Javad Akbari Torkestani and Mohammad Reza Meybodi, "A mobility-based Cluster Formation Algorithm for Wireless Mobile ad-hoc Networks," in Cluster Computing, 2011.

[13] Thomas Watteyne and Antonella Molinaro, "From MANET To IETF ROLL Standardization: A Paradigm Shift in WSN Routing Protocols," in IEEE Communications Surveys and Tutorials, vol.13, no. 4, 2011.

[14] Hui Cheng and Shengxiang Yang "Genetic Algorithms with Elitism-based Immigrants for Dynamic Load Balanced Clustering Problem in Mobile Ad Hoc Networks," IEEE, 2011.

[15] EfiDror and Chen Avin et al "Fast Randomized Algorithm for Hierarchical Clustering in Vehicular Ad-
Hoc Networks," in IEEE Ad-hoc Networking Workshop, June 2011.

[16] Sheetal Mehta and Priyanka Sharma "A Survey on Various Cluster Head Election Algorithms for Manet," in International Conference on Current Trends in Technology, pp. 08-10, December 2011.

[17] Evandro de Souza and Ioanis Nikolaidis, "A New Aggregate Local Mobility (ALM) Clustering Algorithm for VANETs," in IEEE International Conference on Communications, May 2010.

[18] Soumyabrata Talapatra and Alake Roy "Mobility Based Cluster Head Selection Algorithm for Mobile Ad-Hoc Network," in International Journal of Computer Network and Information Security, pp.42-49, 2014.

[19] HongjieJia, Shifei Ding and Hong Zhu "A Feature Weighted Spectral Clustering Algorithm Based on Knowledge Entropy," in Journal of Software, vol. 8, no. 5, May 2013.

[20] S. Muthuramalingam and R. Raja Ram, "A Dynamic Clustering Algorithm for MANETs by modifying Weighted Clustering Algorithm with Mobility Prediction", in International Journal of Computer and Electrical Engineering, vol.2, no. 4, August 2010. 